\title{
Considerações sobre fixadores externos sob a perspectiva do paciente
}

\author{
Considerations about external fixators from the patients perspective \\ Consideraciones sobre fijadores externos desde la perspectiva del paciente \\ Eli Ávila SOUZA JÚNIOR ${ }^{1}$ \\ Pedro Henrique Silva CAMPOS ${ }^{2}$ \\ Rafael Luiz de Pinho Teixeira MOURÃO ${ }^{3}$ \\ Daniel Soares BAUMFELD ${ }^{4}$ \\ Túlio Vinícius de Oliveira CAMPOS ${ }^{5}$ \\ Marco Antônio Percope de ANDRADE ${ }^{6}$
}

Departamento do Aparelho Locomotor, Faculdade de Medicina da Universidade Federal de Minas Gerais-UFMG, 30130-100 Belo Horizonte-MG, Brasil

\begin{abstract}
Resumo
Introdução: Dentre as medidas terapêuticas impostas nas fraturas, os fixadores externos são dispositivos frequentemente usados, principalmente em hospitais referência de trauma. A fixação externa pode gerar desfiguramento ao corpo humano e conduzir a um grau de invalidez. Ocorre um inevitável insulto a imagem corporal e causa rompimento do relacionamento pessoal e social, podendo gerar depressão e retração. Objetivos: Avaliar as considerações de pacientes atendidos em um hospital referência de trauma sobre o uso dos fixadores externos. Métodos: Estudo qualitativo e descritivo realizado no Hospital Universitário Risoleta Tolentino Neves, Belo Horizonte, com 20 pacientes em uso de fixadores externos. Para a análise e apresentação dos resultados utilizou-se o Discurso do Sujeito Coletivo, redigido na primeira pessoa do singular, composto por expressões chaves que tiveram as mesmas ideias centrais e mesma ancoragem.

Resultados: Quanto à função dos fixadores externos, os pacientes demonstraram conhecimento, exibindo como ideias centrais, sua atuação como tratamento definitivo, tratamento temporário, uso em casos complexos, e controle da dor. Ainda, quanto às suas impressões sobre o uso dos mesmos, foram mencionadas considerações negativas como a ocorrência de dor e incômodo; referências sobre as condições estéticas; todavia, a maioria manifestou otimismo sobre o uso do mesmo, exibindo compreensão sobre o seu benefício. Conclusão: A despeito do escasso conteúdo na literatura que explora as percepções dos pacientes sobre os fixadores externos, notamos que a maioria tem conhecimento sobre a função do mesmo, manifestando, sobretudo, otimismo em relação ao seu uso.
\end{abstract}

Descritores: Pesquisa Qualitativa; Bioética; Qualidade de Vida; Fixação de Fratura.

\section{Abstract}

Introduction: Among the therapeutic measures imposed on fractures, external fixators are frequently used devices, especially in hospitals referenced in trauma. External fixation involves considerable disfigurement of the human body and leads to a degree of disability. There is an inevitable insult to body image and it causes disruption of personal and social relationships and this can lead to withdrawal and depression. Objectives: To evaluate the considerations of patients treating in a hospital referenced of trauma about the use of external fixators. Methods: A qualitative and descriptive study performed at Hospital Universitário Risoleta Tolentino Neves, Belo Horizonte, with 20 patients using external fixators. For the analysis and presentation of the results we used the Collective Subject Discourse, written in the first person singular, composed of key expressions that had the same central ideas and same anchorage. Results: Regarding the function of the external fixators, the patients demonstrated knowledge, displaying as central ideas, their performance as definitive treatment, temporary treatment, use in complex cases, and pain control. Still, as for their impressions about their use, negative considerations were mentioned such as the occurrence of pain and discomfort; references on aesthetic conditions; however, most expressed optimism about the use of the same, showing understanding about its benefit. Conclusion: In spite of the scarce content in the literature that explores the patients' perceptions about the external fixators we noticed that the majority has knowledge about the function of the same, manifesting, above all, optimism in relation to its use.

Descriptors: Qualitative Research; Bioethics; Quality of Life; Fracture Fixation.

\section{Resumen}

Introducción: Entre las medidas terapéuticas impuestas en las fracturas, los fijadores externos son dispositivos frecuentemente usados, principalmente en hospitales referencia de trauma. La fijación externa puede generar desfiguración al cuerpo humano y conducir a un grado de invalidez. Se produce un inevitable insulto a la imagen corporal y causa rompimiento de la relación personal y social, pudiendo generar depresión y retracción. Objetivos: Evaluar las consideraciones de pacientes atendidos en un hospital referencia de trauma sobre el uso de los fijadores externos. Métodos: Estudio cualitativo y descriptivo realizado en el Hospital Universitario Risoleta Tolentino Neves, Belo Horizonte, con 20 pacientes en uso de fijadores externos. Para el análisis y presentación de los resultados se utilizó el Discurso del Sujeto Colectivo, redactado en la primera persona del singular, compuesto por expresiones claves que tuvieron las mismas ideas centrales y el mismo anclaje. Resultados: En cuanto a la función de los fijadores externos, los pacientes demostraron conocimiento, exhibiendo como ideas centrales, su actuación como tratamiento definitivo, tratamiento temporal, uso en casos complejos, y control del dolor. En cuanto a sus impresiones sobre el uso de los mismos, se mencionaron consideraciones negativas como la ocurrencia de dolor e incomodidad; referencias sobre las condiciones estéticas; sin embargo, la mayoría manifestó optimismo sobre el uso del mismo, mostrando comprensión sobre su beneficio. Conclusión: A pesar del escaso contenido en la literatura que explora las percepciones de los pacientes sobre los fijadores externos, notamos que la mayoría tiene conocimiento sobre la función del mismo, manifestando, sobre todo, optimismo en relación a su uso. Palabras clave: Investigación cualitativa; bioética; calidad de vida; fijación de fracturas

Descriptores: Investigación Cualitativa; Bioética; Calidad de Vida; Fijación de Fractura.

\section{INTRODUÇÃO}

As hospitalizações no Brasil ocasionadas por causas externas envolvendo traumatismos múltiplos, principalmente dos membros inferiores, são um cenário bastante frequente, algo de destaque em países em desenvolvimento. Entre os fatores etiológicos do trauma, os acidentes de trânsito somam o maior percentual de lesões, seguidos de quedas, agressões e lesões autoprovocadas ${ }^{1}$.
Dentre as diretrizes terapêuticas preconizadas nas fraturas, os fixadores externos são dispositivos frequentemente usados. São suficientemente rígidos para manter o alinhamento, facilitam a sustentação do peso, são adaptáveis a uma ampla variedade de condições de traumas e pacientes, além de permitirem tratamento ambulatorial e retorno breve do paciente às atividades cotidianas ${ }^{2-4}$. 
O paciente com fixador externo necessita de uma série de cuidados: manter os pontos cortantes cobertos para previnir lesões, elevar o membro para reduzir edema, monitorar o estado neurovascular da extremidade, avaliar com frequência cada pino para detectar sinal de infecção o mais precoce possível, além de autocuidado para com o dispositivo, que fará parte da vida do paciente por um determinado período ${ }^{5,6}$.

Os eventos traumáticos podem causar deficiências e incapacidades que interferem na capacidade das vítimas sobreviventes cumprirem tarefas, assim como na qualidade de suas vidas. Muitas vezes, os estudos abordam o problema sob o aspecto da mortalidade e/ou demanda aos serviços hospitalares, sendo que são escassas as referências à questão das sequelas, dependências, como também a qualidade de vida após o evento ${ }^{7}$.

Os fixadores externos podem desencadear reações e sentimentos de sofrimento e alterar a imagem corporal da pessoa. Habituar-se a uma estrutura que modifica sua forma física e altera sua autoimagem, tornando-a foco de atenção e curiosidade é um desafio. Os relatos de pacientes ortopédicos vitimados por acidentes rodoviários em Cingapura indicaram, após análise qualitativa, que independentemente da causa e da gravidade dos ferimentos, um evento traumático força a vítima a rever sua vida, altera suas atitudes e causa perdas socioeconômicas ${ }^{8}$.

A área médica se apoderou da expressão qualidade de vida, dando-lhe a conotação de conquista de conforto físico e psicológico, em situações em que o sofrimento dos enfermos pudesse caminhar na direção do bem-estar ou da cura de sinais e sintomas. Nessas circunstâncias, o termo "qualidade de vida em saúde" é mais apropriado, ao se considerar a dicotomia saúde x doença ${ }^{9}$.

Portanto, diante dessa tendência humanizada em promover a "qualidade de vida em saúde" em todas as áreas médicas, tornou-se imperioso estender esse conceito aos hospitais de trauma, e dentro da nossa realidade, conhecer as impressões de nossos pacientes sobre o uso dos fixadores externos, objetivando, assim, conciliar um tratamento efetivo com a otimização dos aspectos psicossociais, e excelência no atendimento.

\section{MATERIAL E MÉTODO}

A Teoria das Representações Sociais (TRS) foi o referencial metodológico adotado, baseado na natureza qualitativa desse estudo. Esta apreende os aspectos mais subjetivos que permeiam as questões da área da saúde. Para conhecer e descrever as considerações dos pacientes sobre o uso dos fixadores externos, sob o referencial das RS, o Discurso do Sujeito Coletivo (DSC) foi o método escolhido por permitir a aproximação com o aproximação com o fenômeno em estudo.

A entrevista individual foi realizada com duas questões semi-estruturadas, após assinatura do termo de consentimento livre e esclarecido (TCLE), obedecendo-se à resolução n. ${ }^{\circ}$ 466/12 do Conselho Nacional de Saúde, que trata de pesquisa envolvendo seres humanos, sendo que as questões éticas deste trabalho foram orientadas pelas suas diretrizes e levadas, pelo investigador, ao conhecimento dos sujeitos da pesquisa. A obtenção do TCLE permitiu que o sujeito da pesquisa compreendesse os procedimentos, riscos, e benefícios, garantindo sua autonomia.

O estudo, realizado no período de 01 de abril de 2017 a 01 de dezembro de 2017, teve como sujeitos 20 pacientes em uso de fixador externo, que estiveram durante esse período internados na enfermaria de ortopedia do Hospital Universitário Risoleta Tolentino Neves, Belo Horizonte, MG.

As entrevistas investigaram as considerações dos pacientes sobre o uso dos fixadores externos, em relação a sua função e suas impressões individuais sobre os mesmos. Cada entrevista foi gravada e depois transcrita para análise. O Discurso do Sujeito Coletivo (DSC), redigido na primeira pessoa do singular, composto por expressões chaves $(\mathrm{ECH})$ que tiveram as mesmas ideias centrais (IC) e mesma ancoragem (AC), foi utilizado para a interpretação dos dados e apresentação dos resultados. A ordem das seguintes etapas foi seguida: 1) as respostas foram ouvidas várias vezes e apenas após o entendimento da ideia geral foram transcritas; 2) todas as respostas dos entrevistados foram lidas e após leitura separada das respostas para a questão analisada; 3) transcrição das respostas para a questão 1 sendo marcadas as ECH em itálico e indicadas as IC, que representaram a descrição das ECH e não a sua interpretação. Todas as questões seguiram o mesmo procedimento; 4) cada ideia central foi transcrita individualmente com respectiva $\mathrm{ECH}$; 5) agrupamento das IC e das frequências de ideias. Por fim, construção dos DSC separadamente de cada ideia central, com respectiva ECH.

\section{RESULTADOS}

A Tabela 1 exibe a caracterização da amostra por sexo, mecanismo de lesão e região do fixador.

Quando abordados com a primeira pergunta (para você, qual a função desse fixador externo que está usando), a ideia central referida por $60 \%$ dos entrevistados foi estabilidade e tratamento da fratura, sendo que $20 \%$ referiram seu uso para casos complexos, $15 \%$ citaram sua função temporária e 1 paciente (5\% da amostra) não sabia referir a função do mesmo.

As ideias centrais (IC), palavras-chave e discurso do sujeito coletivo para a primeira abordagem encontram-se listados abaixo: 
IC: Estabilidade e tratamento da fratura-60\%

DSC: O fixador tem como função alinhar e modelar uma fratura que ficou exposta(...) A aparelhagem externa já indica o sentido dos ossos, o que permite, como eu disse, o retorno do membro a forma original(...) Ele serve para fixar o osso, o qual sem ele, não ficaria no lugar(...)Funciona para permitir a fixação de osso com osso. Ele alinha e da liga, o que facilita do osso de poder colar (...)Não tinha, para mim, outra maneira de juntar meus ossos, se não fosse pelo uso do fixador externo. $\mathrm{O}$ fixador externo foi colocado para não deslocar o osso quebrado, mantendo-o sempre no lugar, estagnado, mesmo comigo podendo ainda mexer a perna (joelho e tornozelo).

\section{IC: Casos complexos - 20\%}

DSC: Ele tem como função a capacidade de unir os ossos, principalmente nesses casos, como o meu, de fraturas complicadas(...) Achei que meu pé tinha sido amputado, quando eu puxei o forro e vi o fixador, fiquei muito feliz, eu acho que meu pé só está aqui pelo fixador, sem ele não teria visto meu pé novamente(...)Como meu dedo foi quebrado em várias partes, ele foi colocado para manter as partes juntas e colar o mais cedo possível.

\section{IC : Tratamento temporário - $15 \%$}

DSC: Está juntando meu osso de novo, eu creio que está fazendo a transição para depois colocar a barra interna, pois como o osso quebrou muito não podia fazer direto(...)Acredito que é para preparar minha estrutura óssea para receber uma nova estrutura, porque eu quebrei muito meu osso;eu fico imaginando se caso eu estivesse com o definitivo, eu não estaria melhor(...).Tinha que ter o fixador primeiro para depois fazer a cirurgia final.

IC: Não sei-5\%

DSC: Não sei direito qual a finalidade dele.

$\mathrm{Na}$ segunda abordagem (qual a sua impressão em relação ao uso do fixador externo) $70 \%$ dos entrevistados referiram como ideia central desconforto e dor, porém $55 \%$ mencionaram otimismo sobre seu uso como ideia central. 15\% enfatizaram sobre seu aspecto estético, e 1 paciente (5\%) não soube mencionar.

As ideias centrais (IC), palavras-chave e discurso do sujeito coletivo (DSC) para a segunda abordagem encontram-se listados abaixo:

\section{IC: Desconforto e dor $-70 \%$}

DSC: Ele incomoda um pouco, dá um peso na minha perna(...) Eu achei ele incômodo porque ele batia na minha outra perna, principalmente nos primeiros dias, até eu me acostumar(...) Ele também me atrapalhou a dormir, pois eu não conseguia virar para o lado dele(...)Ele é bastante incômodo, me atrapalha a dormir, não me deixa virar e, às vezes, bate no outro pé(...) Atrapalha muito, acho ele muito pesado(...) Poderia ser um pouco menor, o tamanho está um pouco desconfortável, para locomover na cama, por exemplo, fica ruim(...)Ele atrapalha para tomar banho, vestir roupa e dormir(...) Machuca muito, sinto muita $\operatorname{dor}(\ldots)$

\section{IC: Otimismo -55\%}

DSC: Eu acho que a partir dele foi possível voltar minha perna para o lugar, por isso foi bastante legal(...) Ele me ajuda a ter mais cuidado a não esbarrar ou forçar a região da fratura. E eu acredito que ele vai me ajudar muito(...)Eu acho que ele vai ajudar bastante, sendo capaz de manter o osso no lugar até ele colar(...) Está ajudando muito a melhorar as bolhas. Eu acho que ele funciona muito bem, conseguiu unir meus ossos em pouco tempo, o que me deu firmeza, me permitiu sentar e, com toda certeza, me permitirá deambular novamente(...)Tenho uma expectativa de que será muito bom para mim e eu preciso dele(...) Tenho a esperança de que será benéfico. Não é confortável, mas acho que seja bastante necessário(...)Incomoda um pouco, mas temos que usar para melhor resultado. Tenho fé nele(...)Minha impressão é que vai dá tudo certo, estou confiando no tratamento(...)

\section{IC: Impressão estética - $15 \%$}

DSC: Ele até é estético, de cor bonita, na moda(...) A aparência feia dele não é problema para $\operatorname{mim}(. .$.$) Ele$ não é muito bonito não, mas acredito que ele me ajudou bastante(...)Horrível a questão visual do mesmo $(. .$.

\section{IC: Não tenho $-5 \%$}

DSC: Não tenho impressão de nada, não sabia direito qual a finalidade dele, mas tecnicamente está bem estabelecido.

Tabela 1. Caracterização da amostra por sexo, mecanismo de lesão e região do fixador

\begin{tabular}{clll}
\hline Sujeito & \multicolumn{1}{c}{ Sexo } & \multicolumn{1}{c}{$\begin{array}{c}\text { Mecanismo de } \\
\text { lesão }\end{array}$} & \multicolumn{1}{c}{$\begin{array}{c}\text { Região do } \\
\text { fixador }\end{array}$} \\
\hline 1 & Feminino & Acidente de moto & Tornozelo \\
2 & Feminino & Queda de altura & Punho \\
3 & Feminino & Queda de altura & Tornozelo \\
4 & Feminino & Queda de altura & Tornozelo \\
5 & Feminino & Queda de altura & Tornozelo \\
6 & Masculino & Acidente de moto & Tíbia \\
7 & Masculino & Acidente de moto & Tíbia \\
8 & Masculino & Projétil de fogo & Úmero \\
9 & Masculino & Queda de altura & Tornozelo \\
10 & Masculino & Acidente de moto & Úmero \\
11 & Masculino & Acidente de moto & Tornozelo \\
12 & Masculino & Acidente de moto & Tíbia \\
13 & Masculino & Projétil de fogo & Fêmur \\
14 & Masculino & Acidente de moto & Tíbia \\
15 & Masculino & Acidente de moto & Fêmur \\
16 & Masculino & Acidente de moto & Tíbia \\
17 & Masculino & Projétil de fogo & Tíbia \\
18 & Masculino & Queda de altura & Tíbia \\
19 & Masculino & Acidente de moto & Pelve \\
20 & Masculino & Projétil de fogo & Tíbia \\
\hline Fon & &
\end{tabular}

Fonte: dados da pesquisa. 


\section{DISCUSSÃO}

Para Minayo et al..$^{10}$, a pesquisa qualitativa responde às questões particulares, considerando como sujeito de estudo pessoas pertencentes a um grupo e com uma determinada condição social, com universo de significados, valores, crenças e atitudes. A pesquisa exploratória é realizada em área na qual há pouco conhecimento acumulado e sistematizado, constituindo-se na primeira etapa de uma investigação mais ampla, e é desenvolvida quando o tema é pouco explorado. Por sua natureza de sondagem, não comporta hipóteses que, todavia, podem surgir durante ou ao final da pesquisa ${ }^{10}$.

A fixação externa tem por função contatar os fragmentos ósseos diretamente e reduzir o movimento no foco da fratura, acelerando a consolidação óssea ${ }^{11-13}$. A versatilidade do fixador externo pode ser notada nas respostas de nossas entrevistas, os quais, quando indagados sobre a função do fixador externo, referiram como ideias centrais: garantia de estabilidade e tratamento da fratura, estabilização temporária e controle de danos em casos complexos.

A capacidade de fixação rígida e alinhamento das fraturas torna o fixador externo consagrado para tratamento de lesões graves dos tecidos moles. Como há maiores chances de infecção em pessoas com fratura aberta, em geral a fixação externa é muito bem indicada ${ }^{14}$. Quando indagados sobre a função do fixador externo, uma parcela dos entrevistados manifestou o conhecimento acima expresso, sobre o uso de fixador externo para controle de danos. Trecho como: "Ele tem como função a capacidade de unir os ossos, principalmente nesses casos, como o meu, de fraturas complicadas", exemplifica isso.

Fatores clínicos, funcionais, sequelas, aspectos psicológicos, dor residual, características sociodemográficas do paciente, e a natureza da lesão e da condução do tratamento, podem exercer uma influência significativa na forma como a vida será vivida daquele momento em diante ${ }^{15}$. Em nosso trabalho, a maior parte dos entrevistados referiu otimismo como ideia central a serem indagados sobre a impressão gerada pelo uso do fixador externo. Apesar dos problemas relacionados a ele citados, como dor, incômodo (desconforto), má aparência estética, notou-se que os pacientes têm consciência sobre o benefício do mesmo, como citado: "Não é confortável, mas acho que seja bastante necessário".

Mckee et al. ${ }^{16}$, em 1998, avaliaram a qualidade de vida de 22 pacientes que usaram o fixador externo de Ilizarov. Os autores observaram que a qualidade de vida estava mais comprometida nesses pacientes do que em alguma outra doença, e que o maior comprometimento abrangia os aspectos físicos, seguido pelos aspectos emocionais, sendo difícil o processo de aceitação psicológica do aparelho. Em nosso estudo, achado compatível foi notado. Grande parcela dos pacientes referiu sensações de incômodo pelo uso do fixador externo. Dificuldades manifestadas como dor, peso, dificuldade para locomover e sono afetado também foram observadas.

Os aspectos psicossociais relacionados à fixação externa foram investigados por $\operatorname{Limb}^{17} \mathrm{em}$ 2003, que destacou o reduzido número de pesquisas que avaliam o cuidado da pessoa com esse dispositivo. Exemplos de descrições para o aparelho compreendem: "terrível e uma forma amigável de tortura", "um aparato de metal que está fora do corpo" ou "grotesco e doloroso". A convivência de pacientes com dispositivos pélvicos caracterizou falas de "mutilação e anormalidade", destacando a alteração na imagem corporal. Nosso estudo contrasta com esse achado em relação à impressão estética. Dos 20 pacientes entrevistados, 4 fizeram referências em relação ao aspecto do fixador externo; 2 referiram imagem negativa do dispositivo, enquanto 2 manifestaram irrelevância sobre o a questão estética.

\section{CONCLUSÃO}

Quanto à função dos fixadores externos, os pacientes, de forma geral demonstraram conhecimento, exibindo como ideias centrais sua atuação como tratamento definitivo, tratamento temporário, uso em casos complexos e controle da dor. Ainda, em relação às suas percepções sobre o uso dos mesmos, a despeito das considerações negativas como a ocorrência de dor, incômodo e condições estéticas, a maioria dos pacientes mostrou-se otimista quanto ao uso do fixador externo, reconhecendo seu valor benéfico.

\section{REFERÊNCIAS}

1. Gawryszewski VP, Koizumi MS, Mello JMHP. As causas externas no Brasil no ano 2000: comparando a mortalidade e a morbidade. Cad Saúde Pública. 2004; 20(4):995-1003.

2. Berhens F. Fixação interna. In: Muller ME, Allgöwer M, Schneider R, Willenegger H (eds). Manual de osteossíntese: técnicas recomendadas pelos grupos AO-ASIF. 3. ed. São Paulo: Manole; 1993. p.367-410.

3. Meeker MH, Rothrock JC. Alexander: cuidados de enfermagem ao paciente cirúrgico. 10. ed. Rio de Janeiro: Guanabara Koogan; 1995.

4. Smeltzer SC, Bare BG. Brunner \& Suddarth: tratado de enfermagem medico-cirúrgica. 8. ed. Rio de Janeiro: Guanabara Koogan; 1998.

5. Ventura MF, Faro ACM, Onoe EKN, Utimura M. Enfermagem ortopédica. São Paulo: Ícone; 1996.

6. Tashiro MTO, Souza MF, Oliveira SD. Autocuidado no tratamento pelo método de Ilizarov: um estudo de caso. R Bras Enferm. 1995; 48(1):46-50.

7. Oliveira NLB, Sousa RMC. Diagnóstico de 
lesões e qualidade de vida de motociclistas vítimas de acidentes de trânsito. Rev Latino-Am de Enfermagem. 2003; 11(6):749-56.

8. Tan KL, Lim LM, Chiu LH. Orthopaedic patients' experience of motor vehicle accident in Singapore. Int Nurs Rev. 2008; 55(1):110-16.

9. Santy J. Nursing the patient with an external fixator. Nurs Stand. 2000; 14(31):47-52.

10. Minayo MCS, Hartz ZMA, Buss PM. Qualidade de vida e saúde: um debate necessário. Ciênc saúde coletiva. 2000; 5(1):7-18.

11. Berhens F. Fixação interna. In: Muller ME, Allgöwer M, Schneider R, Willenegger H (eds). Manual de osteossíntese: técnicas recomendadas pelosgrupos AO-ASIF. 3. ed. São Paulo: Manole; 1993. p.367-410.

12. Meeker MH, Rothrock JC. Alexander: cuidados de enfermagem aopaciente cirúrgico. 10. ed. Rio de Janeiro: Guanabara Koogan; 1995.

13. Smeltzer SC, Bare BG. Brunner \& Suddarth: tratado de enfermagem medico-cirúrgica. $8^{\mathrm{a}}$ ed. Rio de Janeiro: Guanabara Koogan; 1998.

14. Rothrock JC. Alexander: cuidados de enfermagem ao paciente cirúrgico. Rio de Janeiro: Elsevier; 2007.

15. O'toole RV, Castillo RC, Pollak AN, MacKenzie EJ, Bosse MJ, LEAP Study Group. Determinants of patient satisfaction after severe lowerextremity injuries. J Bone Joint Surg Am. 2008; 90(6):1206-11.

16. Mckee, M.D.; Yoo D.; Schemitsch, E.H. Health status after Ilizarov reconstruction of posttraumatic lower-limb deformity. J Bone Joint Surg. v.80, n.2, p.360-5, 1998.

17. Limb M. Psychosocial issues relating to external fixation of fractures. Nurs Times. 2003;99(44)2830.

\section{CONFLITO DE INTERESSES}

Os autores declaram não haver conflitos de interesse.

\section{AUTOR PARA CORRESPONDENCIA}

\section{Eli Ávila Souza Júnior}

elijr42@yahoo.com.br
Submetido em 01/07/2018

Aceito em 12/09/2018 